\title{
Tratamento cirúrgico do megaesôfago no Hospital de Clínicas da UNICAMP - fatores associados a melhores ou a piores resultados
}

\section{Surgical treatment of megaesophagus at UNICAMP Hospital of Clinics - associated factors with better and worse results}

Gustavo Carvalho de Oliveira ${ }^{1}$; Luiz Roberto Lopes, TCBC-SP²; Nelson Adami Andreollo, TCbC-SP3; Nathália da Silva Braga ${ }^{1}$; JoÃo de Souza Coelho Neto ${ }^{4}$

\section{R E S U M O}

\begin{abstract}
Objetivo: Estudar e verificar os possíveis fatores associados a melhores ou a piores resultados cirúrgicos em pacientes submetidos a tratamento cirúrgico para megaesôfago. Métodos: Um estudo retrospectivo analisou dados específicos nos prontuários dos pacientes. Avaliaram-se a ocorrência de complicações no intra ou no pós-operatório e a manutenção de queixas de disfagia que merecesse alguma intervenção após a cirurgia, com o auxílio de um questionário dirigido, estudando possíveis associações a: modalidade cirúrgica adotada, grau da doença, etiologia e presença de outras doenças digestivas. 0 total de pacientes operados foi 417, sendo levantados 390 prontuários. Resultados: Foram 360 cardiomiotomias, 20 esofagectomias e 11 mucosectomias. Sete pacientes saíram da análise por não estarem adequadamente registrados. Conclusão: O tipo de operação é o que mais influi nos resultados cirúrgicos (as cardiomiotomias têm melhor resolução); as complicações são maiores com o aumento do grau do megaesôfago. O tratamento cirúrgico em pacientes com doença chagásica teve resultados piores que nos pacientes com megaesôfago idiopático; a associação de outras doenças do trato digestivo é fator de piora nos resultados pós-cirúrgicos.
\end{abstract}

Descritores: Acalásia esofágica. Doença de Chagas. Esofagopatias. Esôfago. Cirurgia.

\section{INTRODUÇÃO}

O megaesôfago é uma das formas de apresentação clínica da doença de Chagas. Estima-se que ao menos $4 \%$ dos chagásicos brasileiros apresentem essa alteração (cerca de 300mil doentes) $)^{1-3}$. Nele, ocorre destruição de plexos nervosos intramurais do esôfago, redução de peristaltismo ao nível do corpo do órgão e não abertura do esfíncter inferior (acalásia) à deglutição ${ }^{1,4}$. Assim, há incoordenação motora, dilatação e redução de sua capacidade de contração. Admitem-se outras causas para o megaesôfago, nesses casos, chamando-o de Acalásia Idiopátia do Esôfago (AlE) $)^{5,6}$.

O sintoma principal é a disfagia sendo a manifestação que leva o doente a procurar o médico e determina o diagnóstico, sendo geralmente progressiva ${ }^{3,7}$. megaesôfago é graduado de acordo com o diâmetro transverso do órgão, variando de I a IV ${ }^{8}$. Seu tratamento é cirúrgico e essa classificação auxilia a escolha da operação $0^{3,9}$.

Neste trabalho, procurou-se associar alguns fatores a melhores ou a piores resultados no tratamento cirúrgi- co do megaesôfago, nos pacientes operados no Hospital de Clínicas da Unicamp, de 1989 a 2005.

\section{MÉTODOS}

Para avaliar os resultados cirúrgicos dos pacientes operados por megaesôfago desde 1989 até 2005, foram levantados dados sobre complicações intra ou pós-operatórias, se o doente se manteve com queixas de disfagia relevante após a cirurgia (necessitando de dilatações ou novas intervenções cirúrgicas, principalmente, ou insatisfação manifesta), com pelo menos um ano de acompanhamento.

Tais dados foram coletados em levantamento retrospectivo dos prontuários dos pacientes operados por megaesôfago a partir de 1989 até 2005 no HC/Unicamp. Uma ficha/questionário organizou os dados dos atendidos, para facilitar a compilação das informações obtidas.

O total de pacientes operados no período foi de 417; destes, foram levantados 390 prontuários em cerca de oito meses, junto ao SAM (Serviço de Arquivo Médico)

Trabalho realizado na Universidade Estadual de Campinas - UNICAMP- SP-BR.

1. Acadêmico de Medicina da Faculdade de Ciências Médicas da Universidade Estadual de Campinas - SP-BR; 2. Professor Livre-Docente da Disciplina de Moléstias do Aparelho Digestivo do Departamento de Cirurgia da Faculdade de Ciências Médicas da Universidade Estadual de Campinas - SP-BR; 3. Professor Titular da Disciplina de Moléstias do Aparelho Digestivo do Departamento de Cirurgia da Faculdade de Ciências Médicas da Universidade Estadual de Campinas - SP-BR; 4. Médico Assistente da Disciplina de Moléstias do Aparelho Digestivo do Departamento de Cirurgia da Faculdade de Ciências Médicas da Universidade Estadual de Campinas - SP-BR. 
do HC/Unicamp. Vinte e sete prontuários não foram localizados. Os estudados somaram um total de 390, sendo 216 homens $(55,38 \%)$ e 174 mulheres $(44,62 \%)$, com as respectivas médias de idade: 46,5 e 47,7 anos.

Havia 170 homens chagásicos (78,70\%) e 46 portadores da acalásia idiopática (21,30\%). No grupo das mulheres, eram 139 chagásicas $(79,88 \%)$ e 35 não chagásicas $(20,12 \%)$. O total de chagásicos foi 309 $(79,23 \%)$ e de acalásia idopática 81 pacientes $(20,77 \%)$. Além de sexo, idade e etiologia chagásica, os fatores estudados como possivelmente influentes nos resultados cirúrgicos foram: a modalidade cirúrgica adotada, o grau do megaesôfago e a existência ou a ausência de doenças digestivas associadas, como o megacólon, gastrites e esofagites. Para avaliar os bons ou os maus resultados cirúrgicos, foram abordadas as complicações anotadas nas cirurgias e a manutenção da disfagia relevante após a operação.

Para avaliar o grau do megaesôfago, utilizou-se a classificação de Mascarenhas ${ }^{8}$, que vai de I a IV, de acordo com o diâmetro transverso do órgão, sendo: grau l - até $4 \mathrm{~cm}$, grau $I I-$ de $4 \mathrm{~cm}$ a $7 \mathrm{~cm}$, grau $I I I-7 \mathrm{~cm}$ a $10 \mathrm{~cm}$, grau IV - mais de $10 \mathrm{~cm}$.

Os critérios para avaliar a etiologia do megaesôfago foram: exames com técnicas de imunofluorescência ou ELISA com pelo menos duas sorologias negativas, antecedentes epidemiológicos negativos para a doença de Chagas e ausência de doença intestinal associada qualificaram a acalásia como idiopática, enquanto uma sorologia positiva ou antecedente epidemiológico para Chagas aponta para presença de doença de Chagas e, conseqüente megaesôfago chagásico.

A observação de complicações durante ou imediatamente após a cirurgia (perfuração da mucosa esofágica, lesão esplênica, lesão no nervo laríngeo recorrente, derrame pleural, lesão do ducto torácico, fístulas ou estenoses de anastomoses, etc) foram pesquisadas através dos relatos existentes no prontuário dos pacientes.

A análise estatística foi realizada com o teste do Qui-Quadrado. Este trabalho faz parte de projeto aprovado pelo Comitê de Ética em Pesquisa da Faculdade de Ciências Médicas da Universidade Estadual de Campinas - Parecer n 700/2005.

\section{RESULTADOS}

A avaliação dos dados e as comparações percentuais, não mostraram diferenças relevantes entre homens e mulheres, nem de suas médias de idade, sendo assim agrupados conjuntamente.

No total, foram estudadas 360 cardiomiotomias (357 com válvula à Pinotti, uma à Lind, uma à Nissen e uma sem confecção de válvula), uma cirurgia de ThalHatafuku, 20 esofagectomias e 11 mucosectomias.

Seis pacientes saíram do grupo de estudo por não estarem devidamente registradas as suas cirurgias nos prontuários. Também se optou por não avaliar resultados da cirurgia de Thal-Hatafuku por ter sido única e com apenas uma observação, as análises não seriam estatisticamente significativas.

Foram encontradas complicações em 72 cardiomiotomias (20\%), sendo a maioria, 67 (18,6\%) no intraoperatório, todas de menor gravidade. As complicações nas cardiomiotomias foram, em sua maior parte, perfurações acidentais das mucosas (esofágicas, gástricas ou TEG - transição esôfago-gástrica) - 10,2\%, imediatamente suturadas. Houve ainda lesões esplênicas (2\%), com 0,6 $\%$ de esplenectomias, lesões hepáticas (1\%), lesões ou alterações pulmonares/pleurais (1\%) e outras complicações isoladas. O total de cirurgias com dois ou mais complicações foi de apenas três(<1\%).

Em relação às cirurgias para o megaesôfago grau IV tanto nas esofagectomias como nas mucosectomias, o esôfago foi substituído por um tubo gástrico.

Nas mucosectomias, anotaram-se complicações em seis delas (54,5\%), tanto menores ou maiores, sendo que em quatro casos (36,3\%), houve algum tipo de complicação no pós-operatório. Em dois casos (18,2\%), as complicações se limitaram ao intra-operatório. Do total de complicações, a mais freqüente foi a fístula na anastomose esôfago-gástrica, ocorrendo em três casos $(27,2 \%)$ e em $50 \%$ das cirurgias com complicações. Em um desses casos, houve, também estenose dessa mesma anastomose. Houve, ainda, duas lesões esplênicas (18,2\% das mucosectomias), duas complicações pulmonares $(18,2 \%)$ - pneumotórax e derrame pleural, uma lesão hepática e uma suspeita de lesão do nervo laríngeo-recorrente. O total de cirurgias com duas ou mais complicações foi de cinco (45\%).

Já nas esofagectomias, as complicações são ainda mais freqüentes ocorrendo em 17 delas (85\%), tanto menores ou maiores, sendo que em 11 casos (55\%) com intercorrências no intra-operatório. As complicações mais freqüentes foram as pleurais - derrames e lesões, ocorridas em 12 cirurgias (60\% das cirurgias). Foram observados, ainda, quatro casos de pneumotórax (20\%), três estenoses da anastomose esôfago-gástrica (15\%), três casos de infecção sistêmica no pós-operatório (15\%), duas cirurgias com lesões no ducto torácico (10\%), duas atelectasias (10\%), dois casos de hipotensão intra-operatória controladas (10\%) e duas lesões no fundo gástrico (10\%). Houve casos isolados de lesão do nervo laríngeo, lesão hepática, da vesícula biliar, fístula da anastomose esôfago-gástrica, lesão do meso do cólon transverso e empiema pleural e sepse. O total de cirurgias com duas ou mais complicações foi de 12 (60\%), tendo-se revelado como a modalidade cirúrgica de maiores riscos.

A análise estatística comparando o índice de complicações nos três tipos de cirurgia mostrou diferença significativa entre elas $(P<0,05)$.

De acordo com o grau do megaesôfago e as complicações encontradas observou-se: grau I= 14\% $(n=72)$; grau $\|=21 \%(n=185)$; grau $\| I=25 \%(n=90)$; grau $I V=67 \%$ $(n=36)$. A análise estatística das complicações entre os di- 
ferentes graus de megaesôfago, mostrou que há uma tendência a maiores taxas de complicações nos graus maiores de megaesôfago $(p<0,05)$.

As figuras 1 a 4 mostram os dados mais relevantes de cada uma das modalidades e suas complicações mais freqüentes.

A respeito da queixa de disfagia, para avaliar o resultado dos diferentes tipos de cirurgia no pós-operatório tardio, por um período mínimo de um ano de acompanhamento, observou-se que 66 pacientes (18\%) submetidos à cirurgia de Heller mantiveram ou tiveram retorno das queixas de disfagia de modo relevante. Dos pacientes operados por mucosectomia, cinco $(45 \%)$ tiveram queixas disfágicas após a cirurgia e em quatro (20\%) dos submeti-

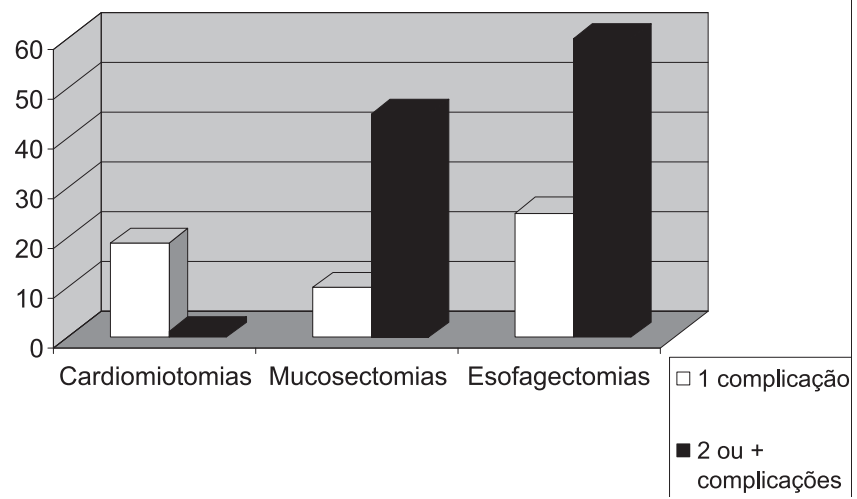

Figura 1 - Número de complicações em cada modalidade cirúrgica (\%).

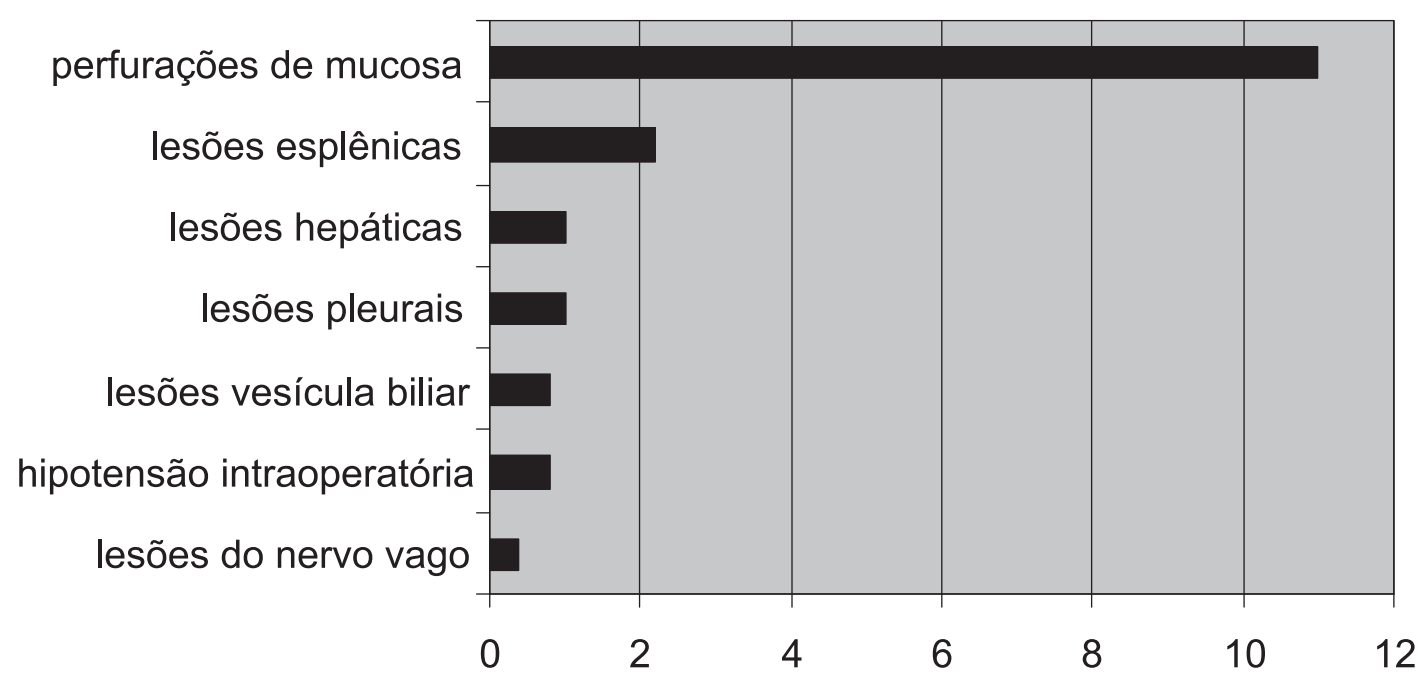

Figura 2 - Cardiomiotomias - complicações frequentes (\%).

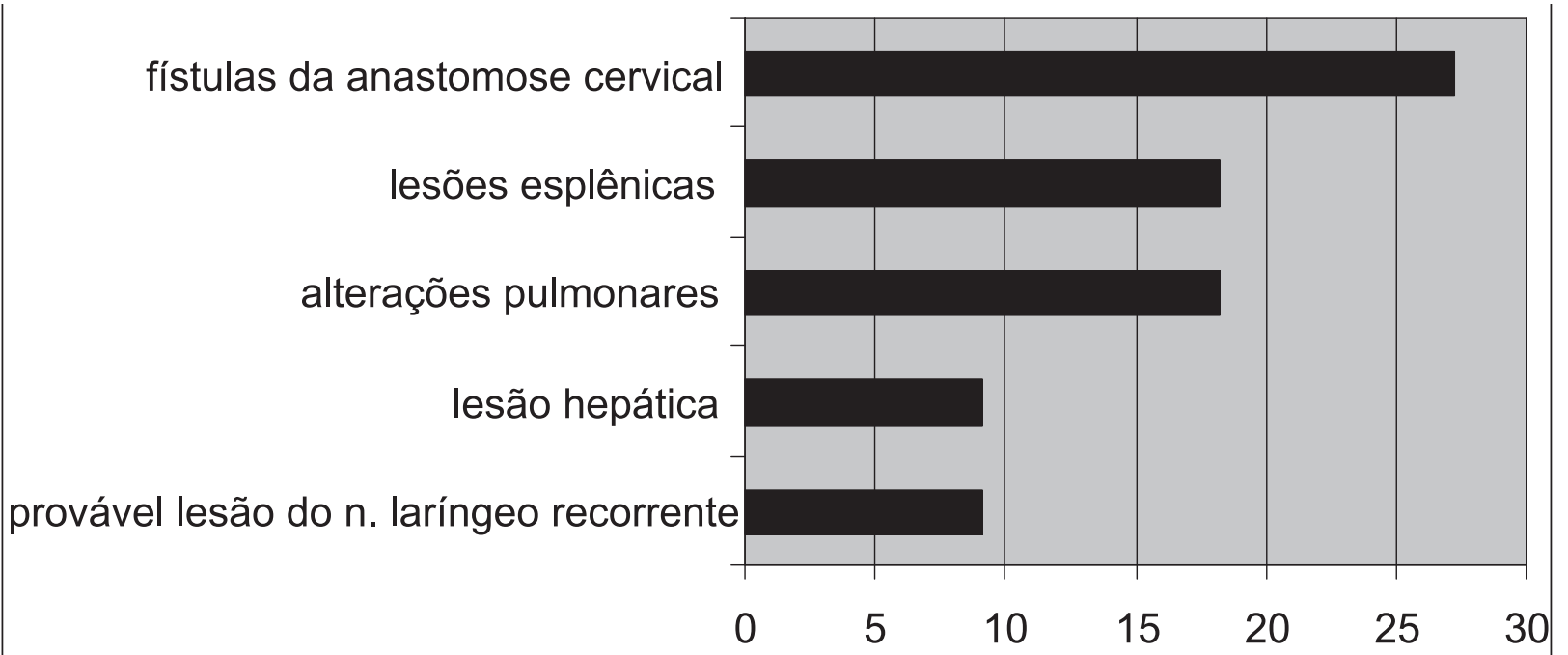

Figura 3 - Mucosectomias - complicações mais frequentes (\%). 


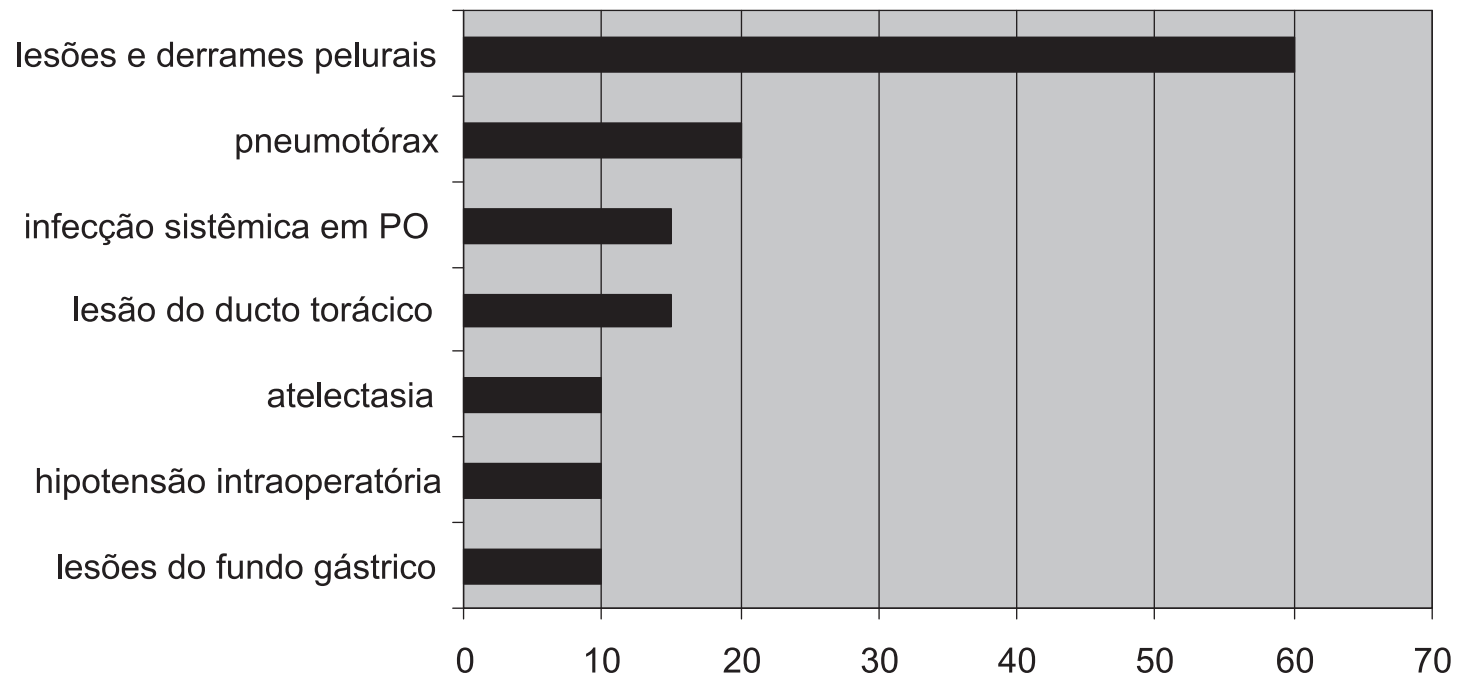

Figura 4 - Esofagectomias - complicações mais frequentes (\%).

dos à esofagectomia em um momento inicial relacionada à anastomose, sendo tratados por dilatação endoscópica.

De acordo com o grau de megasôfago e as queixas de disfagia relevante no pós-operatório, encontrou-se: grau $\mid=19 \%(n=72)$; grau $\|=15 \%(n=185)$; grau $\|=17 \%$ $(n=90) ;$ grau $I V=25 \%(n=36)$.

Sobre a etiologia do megaesôfago (chagásico ou não chagásico), foram obtidos os seguintes dados:

I-geral. Disfagia relevante mantida após cirurgia: chagásicos=18\% ( $n=299)$; idiopáticos=15\% $(n=84)$.

a) chagásicos - grau $\mid=21 \%(n=48)$, grau $\|=16 \%$ $(n=146)$, grau $I I=19 \%(n=73)$, grau IV=24\% $(n=32)$.

b) idiopáticos - grau l=17\% ( $n=25)$, grau $\|=13 \%$ ( $n=41)$, grau $I I=13 \%(n=17)$. O grau IV idiopático não foi avaliado, porque houve apenas dois casos e o $n$ estudado seria muito pequeno.

A análise estatística procurando relacionar disfagia mantida de modo relevante após cirurgia e diferentes graus de megaesôfago, assim como a relação entre disfagia mantida e etiologia do megaesôfago não mostraram diferenças significativas ( $p>0,05 \%$ ).

Os antecedentes apresentados pelos pacientes operados (o grau do megaesôfago, a sua etiologia e outras doenças digestivas associadas) que, possivelmente, se associariam aos resultados cirúrgicos são mostrados na figura 5 .

O último fator avaliado foi a associação de outras moléstias do trato digestivo e a manutenção de disfagia relevante após a operação. Foram agrupados em quatro grupos das moléstias mais prevalentes (para um $n$ total=384 pacientes).

Os mais prevalentes foram: 1-lesões gástricas (gastrites, úlceras) - 55\%; 2-lesões do esôfago (úlceras, esofagites, Barrett) - 37\%; 3- megacólon - 19\%; 4-lesões duodenais - $12 \%$.

Os pacientes sem nenhuma outra doença digestiva associada tiveram índice de disfagia relevante mantida após a cirurgia de apenas $13 \%(n=78)$. Os que tinham outras doenças do esôfago (identificadas principalmente por endoscopias) tiveram esse índice igual a 23\% $(n=141)$. Pacientes com problemas no estômago tiveram disfagia mantida em 19\% ( $n=210)$ dos casos. Os que apresentavam alterações no esôfago e no estômago voltaram a apresentar disfagia em $25 \%$ dos casos $(n=76)$. Os com alterações em esôfago, estômago e duodeno concomitantes apresentaram índice de 31\% $(n=16)$. O megacólon associado ao megaesôfago teve disfagia no pós-operatório em 22\% dos casos $(n=73)$.

Pacientes com megacólon e outras doenças no esôfago tiveram 37\% $(n=29)$ de resultado ruim na correção da disfagia. O pior grupo foi o que teve megacólon, outras doenças no esôfago e outras doenças no estômago associadas ao megaesôfago, com 47\% $(n=17)$ de queixas de disfagia mantidas após a cirurgia.

A figura 6 mostra os resultados das associações nas diferentes combinações de moléstias.

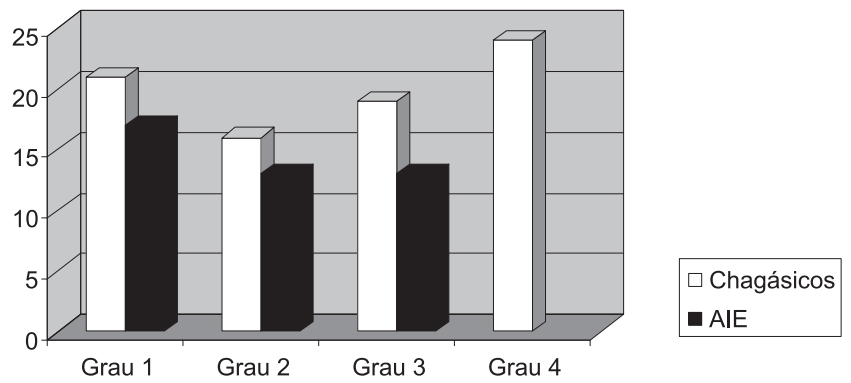

Figura 5: disfagia pós-operatória, segundo grau do megaesôfago e respectiva etiologia (\%)

Figura 5 - Disfagia pós-operatória, segundo grau do megaesôfago e respectiva etiologia (\%). 


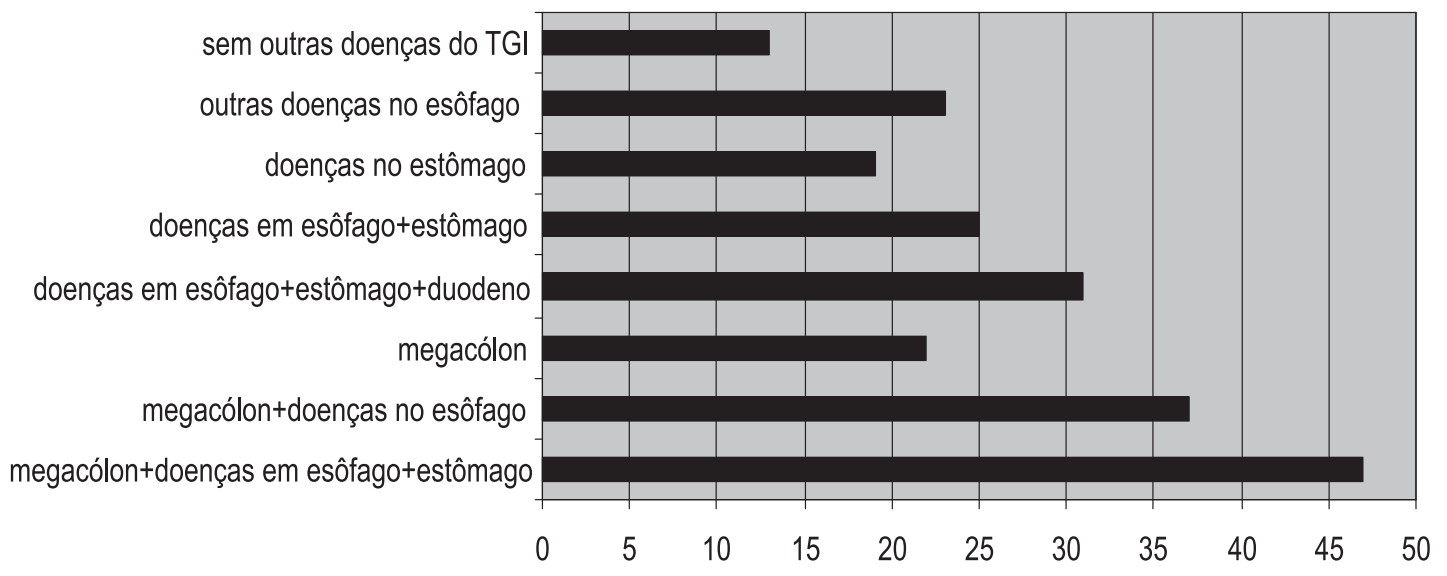

Figura 6 - Manutenção de disfagia grave associada a outras doenças no TGI (\%).

\section{DISCUSSÃO}

Com enfoque nos fatores associados a melhores ou piores resultados após cirurgia para tratamento das diversas formas de apresentação do megaesôfago, quer seja de etiologia chagásica ou não, pode-se observar que a cardiomiotomia é a de melhor resultado e segurança, no quesito presença de complicações, pelo baixo índice de complicações, apesar de a maior parte acontecer no intraoperatório, devidamente resolvidas no momento cirúrgico, - que reforça o bom resultado e a segurança da cardiomiotomia, já encontrado em outros trabalhos nesta década e em anteriores 10-17. Valezi et al., encontraram complicações mais freqüentes, como perfuração de mucosa gástrica ou esofágica em $25 \%$ de pacientes operados por via laparoscópica ${ }^{18}$. Barbosa et al., tiveram 20,2\% de complicações, sendo $12 \%$ de perfurações inadvertidas da mucosa ${ }^{11}$

Nas mucosectomias, observaram-se complicações em $55 \%$ dos pacientes, sendo que dois terços destas ocorreram no pós-operatório. O índice de complicações para este tipo de cirurgia é esperado, principalmente as relacionadas ao pulmão e à anastomose, onde ocorreu fístula em metade dos casos com complicações e uma estenose. Essas complicações com a anastomose devem ser as responsáveis pela queixa de disfagia relevante no pós-operatório, e foram facilmente controladas com dilatações endoscópicas em número variado de sessões. O total de cirurgias com duas ou mais complicações foi de cinco $(45 \%)$, o que é justificado pelo grande porte da cirurgia. Aquino e cols mostraram bons resultados com a mucosectomia ${ }^{19,20}$. Em 60 cirurgias avaliadas, foram 18,3\% de complicações registradas, além de dois óbitos $(3,3 \%)^{20}$.

Quanto às taxas de complicações das esofagectomias ( $85 \%$ ), elas foram mais frequentes do que nas mucosectomias, tanto as que ocorreram no intra-operatório como no pós-operatório. As complicações no tempo operatório foram semelhantes nos dois tipos de cirurgia. As complicações respiratórias foram o dobro na esofagectomia em comparação à mucosectomia, fator possivelmente explicado pela manipulação do espaço mediastinal para dissecção do esôfago, levando ao aumento das complicações locais e sistêmicas, inclusive com dois pacientes $(11 \%)$ tendo apresentado instabilidade no intra-operatório e três (17\%) tiveram infeccção sistêmica. Não houve fístula da anastomose em nenhuma cirurgia e há apenas um relato de estenose. Contudo, ocorreram dois casos de fístula quilosa por lesão do ducto torácico, mais uma vez pelo acesso usado para essa cirurgia. 0 número de cirurgias com duas ou mais complicações nessa modalidade alcançou $60 \%$ delas, maior que nas outras modalidades cirúrgicas.

Por esses números observou-se que a esofagectomia é a cirurgia com o maior índice de complicações e de diferentes tipos de complicações, inclusive as de carácter sistêmico, colocando em maior risco a vida do paciente. Felizmente, não foram observados óbitos em nenhum dos tipos de cirurgia. Tomashich e cols (2003), encontraram complicações precoces no pós-operatório de $39,3 \%$ e letalidade de $13,7 \%{ }^{21}$. Braghetto et al. obtiveram $33,3 \%$ de complicações precoces no pós operatório e 22\% de complicações tardias²2.

$\mathrm{Na}$ avaliação clínica da persistência ou da recorrência do sintoma de disfagia relevante após um período mínimo de um ano de acompanhamento, encontraram-se números semelhantes entre os diferentes tipos de cirurgia: $18 \%$ dos pacientes submetidos à cirurgia de Heller relataram queixas de disfagia relevante. Não houve qualquer correlação entre o paciente ter complicação cirúrgica e de ter disfagia posteriormente. $\mathrm{O}$ índice de disfagia relevante no pós-operatório é normalmente inferior a $20 \%$, em outros estudos científicos ${ }^{11-13,15,23}$.

Dos pacientes operados por mucosectomia, cinco $(45 \%)$ tiveram queixas disfágicas relevantes após a cirurgia. Destes, quatro deles (80\%) também apresentaram complicações cirúrgicas. No grupo sem queixas de disfagia relevante após a cirurgia, apenas um (17\%) também teve complicações cirúrgicas. Avaliando-se o tipo de complicação para os disfágicos no pós-operatório, encontrou-se que três deles tiveram problemas na anastomose esôfago-gástrica, sendo duas fístulas e uma estenose, e o outro teve provável lesão do nervo laríngeo- 
recorrente. Obviamente, tais danos prejudicam diretamente, a deglutição e explicam essa incidência de disfagia relevante no grupo com essas complicações. Todos foram tratados com dilatações endoscópicas alcançandose a resolução do sintoma. Outros estudos sobre resultados da mucosectomia revelam bons resultados, mas os critérios utilizados são diferentes e dificultam uma comparação explícita ${ }^{19,20 .}$

No grupo de pacientes operados por esofagectomia, a disfagia relevante foi observada em quatro pacientes $(20 \%)$, mostrando um bom prognóstico para essa complexa cirurgia. Destes, três também tiveram complicações na cirurgia, não relacionadas à anastomose. Esse resultado demonstra que as complicações na esofagectomia não exercem influência considerável no prognóstico quanto à disfagia, desde que não ocorram problemas com a anastomose. Da mesma maneira, foram tratados com dilatação endoscópica e resolução do sintoma.

Esses dados indicam um índice de complicações cirúrgicas progressivamente maior, de acordo com o crescimento dos graus de megaesôfago. Apesar de o tratamento cirúrgico ser o mesmo nos graus I a III de megaesôfago (cardiomiotomia com fundoplicatura), muito provavelmente o avanço da doença dificulta a cirurgia, seja na manipulação do esôfago e das estruturas próximas no momento cirúrgico. Porém, ao analisar-se a manutenção ou a ausência da disfagia relevante, o resultado é muito parecido nos graus I a III de megaesôfago, o que tende a confirmar o bom resultado da cirurgia de Heller (respectivamente, 19\%, 15\% e $21 \%$ de manutenção). Mesmo com uma resolução um pouco pior nos megaesôfagos de grau IV (tratados com esofagectomia ou mucosectomia), cerca de $25 \%$ de disfagia, a solução desse sintoma foi boa com a dilatação endoscópica de modo geral, corroborando outros trabalhos ${ }^{10,19,20 .}$

Na comparação dos sintomas de disfagia relevante entre as diferentes etiologias (chagásica ou idiopática), não se encontra diferença nos resultados, mostrando que a causa não foi fator de pior ou melhor prognóstico no controle da disfagia.

Especificando-se os graus do megaesôfago e a etiologia, observou-se uma distribuição semelhante que confirma uma tendência de resolução um pouco pior para os pacientes chagásicos, previamente mostrada na figura três. Diferenças entre essas moléstias já foram abordadas em trabalhos anteriores ${ }^{5,6}$.

Quando se avaliou as doenças concomitantes ao megaesôfago, verificou-se que os pacientes que também tinham sintomas de megacólon associados a doenças do esôfago e do estômago, foram os que tiveram as maiores taxas de disfagia no pós-operatório. As demais associações não apresentaram números muito diferentes entre eles.

Apesar de os dados indicarem fortemente uma possível associação entre essas outras doenças do trato digestivo e o resultado pós-operatório menos satisfatório, vale destacar a dificuldade que alguns pacientes apresentam em diferenciar seus sintomas (disfagia, odinofagia, queimação, etc.) e esse dado pode ter sido um pouco sobreestimado. A figura seis já mostrou com clareza esses dados.

O estudo dos fatores associados a melhores ou a piores resultados no tratamento cirúrgico dos pacientes operados de megaesôfago (chagásico ou idiápático) no Hospital de Clínicas da Unicamp entre 1989 e 2005 nos permitiu concluir que: o tipo de operação é o que mais influi nos resultados cirúrgicos: as cardiomiotomias têm melhor resolução; as complicações são maiores com o aumento do grau do megaesôfago, embora isso também pareça refletir o impacto da modalidade cirúrgica (cardiomiotomia, de grau I a grau III; esofagectomia ou mucosectomia no grau IV); a cirurgia em pacientes de etiologia chagásica teve resultados pouco piores que nos pacientes idiopáticos; a associação de outras doenças do trato digestivo é fator de piora nos resultados póscirúrgicos.

\section{A B S T R A C T}

Objectives: To verify some possible factors, which would be associated with better or worse results for surgical treatment of megaesophagus. Methods: There were 417 patients operated on for megaesophagus, but only 390 medical charts were analyzed between 1989 and 2005. The presence of intraoperative and postoperative complications and the maintenance of severe dysphagia complaints were evaluated in a directed questionnaire, studying association with: the surgical approach chosen, megaesophagus degree, etiology and presence of other digestive alterations. Results: There were 360 cardiomiotomies, 20 esophagectomies and 11 mucosectomies. The results indicate that the cardiomiotomy is the safest surgery and the esophagectomy has more complications. The degree of megaesophagus is directed related with the results; more advanced megaesophagus has the worse results. The presence of digestive alterations has also a direct influence with worse results. Conclusion: The most important factor considering the results is the surgery chosen, and the best one were seen with cardiomiotomy. The degree of megaesophagus has also influenced the results. The etiology suggests better results with Chagas disease patients, maybe for the chronic course of this disease. The presence of digestive alteration is a factor which causes worse results, especially if associated with gastritis, esophagitis, megacolon and others.

Key words: Esophageal Achalasia. Chagas Disease. Esophageal diseases. Esophagus. Surgery. 


\section{REFERENCIAS}

1. Silveira AC. O controle da doença de Chagas nos países do cone sul da América. História de uma iniciativa internacional - 1991/2001. Uberaba: Fund Ens \& Pesq Uberaba, 2002.

2. Carrilho RP. Estudo longitudinal de 25 anos da doença de Chagas em Mambaí/Buritinópolis (GO) - Brasil [dissertação]. Brasília (DF): Universidade de Brasília; 2001.

3. Coelho JCU. Aparelho digestivo - Clínica cirúrgica. $2^{a}$ ed. Rio de Janeiro: Medsi; 1996.

4. Pinotti HW. Tratado de clínica cirúrgica do aparelho digestivo. São Paulo: Atheneu; 1994

5. Andreollo NA, Lopes LR, Brandalise NA, Leonardi LS. Acalásia idiopática do esôfago: análise de 25 casos. GED Gastroenterol Endosc Dig. 1996: 15(5):151-5

6. Herbella FA, Oliveira DR, Del Grande JC. Are idiopathic and chagasic achalasia two different diseases? Dig Dis Sci. 2004; 49(3):353-60.

7. Crema E, Cruvinel LAF, Werneck AM, Oliveira RM, Silva AA. Correlação manométrico-radiológica e sua importância no tratamento cirúrgico do megaesôfago chagásico. Rev Soc Bras Med Trop. 2003: 36(6): 665-9

8. Mascarenhas LG, Câmera-Lopes LH, Jurema B, Ferreira-Santos R. Padronização técnica da radiologia do megaesôfago. In: Congresso Brasileiro de Gastroenterologia, 1958, Belo Horizonte. Anais... Belo Horizonte.

9. Ceneviva R, Ferreira-Santos R, dos Santos JS, Mente ED, Sankarankutty AK. Alterações cronológicas do perfil dos pacientes e da modalidade de tratamento cirúrgico do megaesôfago chagásico. Acta Cir Bras. 2002; 17(Supl 3):125-8.

10. Herbella FA, Del Grande JC, Lourenço LG, Mansur NS, Hadda CM. Resultados tardios da operação de Heller associada à fundoplicatura no tratamento do megaesôfago: análise de 83 casos. Rev Assoc Med Bras. 1999; 45(4):317-22.

11. Del Grande JC, Herbella FA, Lourenço LG, Mansur NS, Hadda CM. Resultados imediatos da cardiomiotomia com fundoplicatura no tratamento do megaesôfago: análise de 104 casos. GED Gastroenterol Endosc Dig. 1996; 15(5):156-60.

12. Barbosa H, Barichello AW, Vianna AL, Mendelssonh P, Watanabe LM. Tratamento cirúrgico do megaesôfago chagásico: duas décadas de experiência numa região endêmica. Rev Goiana Med. 1989; 35(1/4):1-23.

13. Halabi M, Soria FJ, Sezin M, Martini R. Tratamiento quirurgico del megaesofago mediante la operacion de Heller. Rev Arg Cir. 1984; 47(6):317-20

14. Andreollo NA, Brandalise NA, Leonardi LS. Megaesôfago incipiente: dilatação ou cirurgia? Rev Assoc Med Bras. 1984; 30(1/2):4-6.

15. Espinoza F, Ize L. Acalasia del esofago: resultado de su tratamiento quirurgico. Rev Gastroenterol Mex. 1983; 48(1):23-9.
16. Ibáñez L, Butte JM, Pimentel F, Escalona A, Pérez G, Crovari F et al. Resultados inmediatos y tardíos de la miotomía de Heller laparoscópica en pacientes con acalasia esofágica. Rev Med Chile. 2007: 135(4):464-72.

17. Pilon B, Teixeira FV, Terrazas JPI, Moreira EP, Pillon EY. Aspectos técnicos da esofagocardiomiotomia com divulsão para o tratamento cirúrgico do megaesôfago chagásico não avançado. Rev Assoc Med Bras. 1998; 44(3):179-84.

18. Valezi AC, Mali Junior J, Marson AC, Brito EM, Souza JCL. Tratamento do megaesôfago chagásico grau II por laparoscopia: experiência em 12 casos. Rev Col Bras Cir. 2004; 31(3):148-53.

19. Aquino JLB, Said MM, Fernandes PR. Avaliação tardia da mucosectomia esofágica com conservação da túnica muscular em pacientes com megaesôfago avançado. Rev Col Bras Cir. 2007; 34(1):9-15.

20. Aquino JLB, Reis Neto JA, Muraro CLP, Camargo JGT. Mucosectomia esofágica no tratamento do megaesôfago avançado: análise de 60 casos. Rev Col Bras Cir. 2000; 27(2):109-16.

21. Tomasich FDS, Valladares GCG, Demarchi VCA, Gagliardi D. Complicações e letalidade hospitalar da esofagectomia com linfadenectomia em dois campos: estudo de 132 casos. Acta Oncol Bras. 2003; 23(1):375-87.

22. Braghetto IM, Herrera Gonzalo C, Patricio BP, Owen KB. Esofagectomía por cirugía mini-invasiva vía toracoscópica o laparoscópica: indicaciones y resultados. Rev Chil Cir. 2005; 57(2):118-26

23. Oliveira GC, Lopes LR, Andreollo NA, Coelho Neto JS. O megaesôfago tratado cirurgicamente: perfil epidemiológico dos pacientes operados no Hospital de Clínicas da Universidade Estadual de Campinas entre 1989 e 2005. Rev Soc Bras Med Trop. 2008; 41(2):183-8.

Recebido em 25/10/2008

Aceito para publicação em 26/12/2008

Conflito de interesse: nenhum

Fonte de financiamento: Fundação de Amparo à Pesquisa do Estado de São Paulo (FAPESP), processo nº6/50122-1.

\section{Como citar este artigo:}

Oliveira GC, Lopes LR, Andreollo NA, Braga NS, Coelho Neto JS. Tratamento cirúrgico do megaesôfago no Hospital de Clínicas da Unicamp fatores associados a melhores ou a piores resultados. Rev Col Bras Cir. [periódico na Internet] 2009; 36(4). Disponível em URL: http:// www.scielo.br/rcbc

\section{Endereço para correspondência:}

Gustavo Carvalho de Oliveira

E-mail: gustavok@fcm.unicamp.br 